\title{
Optimal Bayes Joint Decision and Estimation
}

\author{
X. Rong Li \\ Department of Electrical Engineering, University of New Orleans \\ New Orleans, LA 70148, USA, 504-280-7416, 504-280-3950 (fax), xli@uno.edu
}

\begin{abstract}
Many problems involve joint decision and estimation, where qualities of decision and estimation affect each other. This paper proposes an integrated approach based on a new Bayes risk, which is a generalization of those for decision and estimation separately. Theoretical results of the optimal joint decision and estimation that minimizes the new Bayes risk are presented. The power of the new approach is illustrated by applications in target tracking and classification.
\end{abstract}

Keywords: Estimation, decision, Bayes approach, statistical inference, target tracking, target classification. ${ }^{1}$

\section{INTRODUCTION}

Statistical inference is concerned with two primary problems: decision and estimation. ${ }^{2}$ Essentially, estimation approximates an unknown or random quantity that is continuousvalued, while decision is to choose one from a discrete set of candidates. ${ }^{3}$ Decision in this sense includes detection, discrimination, classification, recognition, identification, selection, association, ranking, hypothesis testing, matching, and other problems over categorical or ordinal data. Parameter estimation, signal, image, or state estimation, prediction, filtering, smoothing, forecasting, regression and other inference problems dealing with numerical data are examples of estimation.

Most parts of the prevailing point estimation theory are confined to the case where estimates are in a metric vector space (e.g., estimation error is largely a distance concept and a sum of estimates is usually a meaningful concept). This is not the case for decision theory (e.g., decision error is almost never a distance and a sum of decisions is a meaningless concept). In other words, the estimate space is usually much more structured than the decision space. However, estimation theory is available for dynamic (e.g., process) as well as static situations, whereas the existing decision theory can only deal with candidates that are invariant. Nevertheless, estimation and decision are closely interrelated. There is no clear boundary between them. In some cases, they are basically the same (e.g., decide or estimate the number of targets).

Although some problems involve decision and/or estimation either alone or separately, good solutions to many other problems require joint decision and estimation (JDE). This is

${ }^{1}$ Research supported in part by ARO grant W911NF-04-1-0274, NASA/LEQSF grant (2001-4)-01, and Navy through Planning Systems Contract \# N68335-05-C-0382.

${ }^{2}$ Sometimes a distinction between inference and decision is maintained in the literature. Decision implies that an action will be taken and thus has an associated cost or risk, but inference does not have this implication. We do not pay attention to this implication in this paper.

${ }^{3}$ This is true in most areas, although in some areas such as optimization, decision is not limited to a discrete set. often the case for problems involving inter-dependent discreteand continuous-valued uncertainties and thus decision and estimation affect each other. This should come as no surprise since decision and estimation rely on the dependence of data upon the discrete uncertainty and the continuous uncertainty, respectively.

The prevailing approach to JDE tries to make the best decision first disregarding estimation and then solve the estimation problem as if the decision were surely correct. This "decision-then-estimation" strategy has several serious flaws. Another strategy is "estimation-then-decision." It would not work well if estimation is significantly dependent on decision or estimation is not secondary. In the general case, a joint approach would be more promising than separate decision and estimation as well as decision then estimation or estimation then decision.

While JDE is an old problem and has been studied before, it has received increasing attention in recent years and efforts have been made to overcome the aforementioned drawbacks (see, e.g., [3], [10], [27]), especially for target inference (see, e.g., [1], [4]-[8], [11]-[17], [21]-[24], [26], [28]). In this paper, we propose an integrated approach to JDE based on a novel Bayes risk as a generalization of those for decision and estimation, respectively. In the optimization theoretic parlance, this approach has the potential of arriving at a globally optimal solution, which is inherently superior in performance to the conventional two-stage optimization strategy or separate decision and estimation, especially for problems where decision and estimation are highly correlated. We show the structure of the optimal procedure in this framework. The power of the proposed framework is illustrated by applications to several difficult JDE problems in target inference.

\section{Joint DECision AND ESTIMATION}

\section{A. The JDE Problem}

Many problems involving both continuous and discrete uncertainties entail joint decision and estimation, where qualities of decision and estimation affect each other. Take ground target tracking and recognition as an example. As Fig. 1 illustrates, here we would like to recognize and track several possibly crossing targets simultaneously using data transmitted from multiple sensors (e.g., imagers and radars). Data are uncertain due to clutter, occlusion, maneuver, sensor resolution, multipaths, etc. The recognition and tracking are joint in that, for example, we may want to destroy the tank, but not the truck, and so an accurate track (i.e., time series of state estimates) 
with a recognition error could be a catastrophe while a poor track with a correct recognition would lead to a miss.

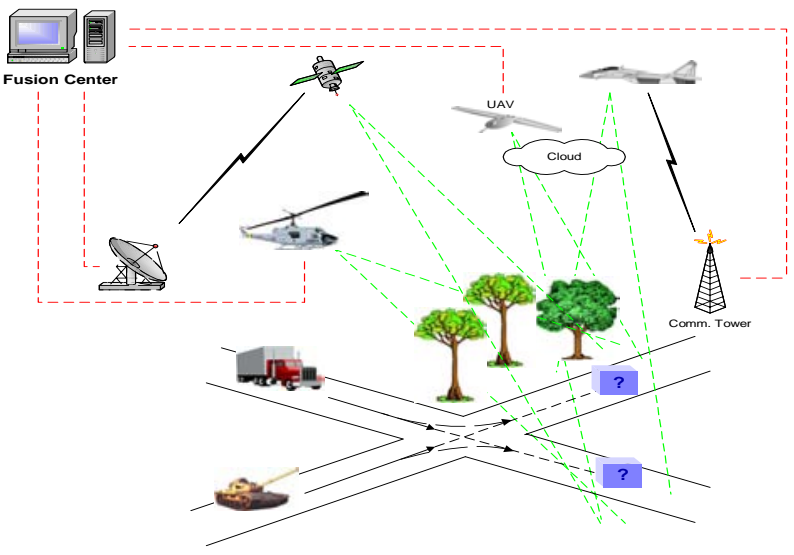

Fig. 1. Joint tracking and recognition of crossing targets.

\section{B. Existing Strategies for Joint Decision and Estimation}

Traditional Strategy. Most conventional solutions to the JDE problems follow the strategy that can be characterized as "decision followed by estimation," "estimation after decision" or simply "decision-then-estimation." It tries to make the best decision first and then do estimation based on the decision made as if it were always the right one. This approach, however, has several serious drawbacks. First, possible decision errors are not accounted for in estimation. Second, decision is done disregarding the quality of the estimation it would lead to (e.g., even if such a decision leads to poor estimation). Although these drawbacks are well perceived, their remedies are hard to come by within this traditional strategy. For example, taking account of decision errors would require estimation in the presence of structural/modal uncertainties (e.g., unknown model-truth mismatch), which is very challenging and is still an open problem in the general setting. Also, no general approach to decision is available that would account for its impact on estimation.

Estimation-then-Decision. Although solutions to most JDE problems follow the traditional decision-then-estimation strategy above, some JDE problems are solved by estimation first and then decision. This is particularly true for composite hypothesis testing. Here the basic idea that underlies all methods is to come up with a simple hypothesis as a surrogate for the original composite hypothesis and then apply the theory to the simple hypotheses. One of the most popular such methods is the (generalized) likelihood ratio test (GLRT), where the original (composite) hypothesis $H_{i}: z \sim f_{i}(z \mid \theta), \theta \in \Theta_{i}$ is replaced by its single (simple) most likely version, $H_{i}$ : $z \sim f_{i}(z)$, obtained by estimation. The hypothesized set $\Theta_{i}$ of possible parameter values is represented by a single value 4 $\hat{\theta}_{i}=\hat{\theta}_{i}^{\mathrm{ML}}=\arg \max _{\theta \in \Theta_{i}} f_{i}(z \mid \theta)$, that is, the maximum likelihood estimate of $\theta$ over $\Theta_{i}$. In other words, the original

\footnotetext{
${ }^{4}$ We prefer readability to rigor. For example, all sup are replaced by max.
}

composite distribution $f_{i}(z \mid \theta), \theta \in \Theta_{i}$ is replaced by the most likely distribution $f_{i}\left(z \mid \hat{\theta}_{i}^{\mathrm{ML}}\right)=\max _{\theta \in \Theta_{i}} f_{i}(z \mid \theta)$. The test is then based on the simple hypotheses $H_{i}: z \sim f_{i}\left(z \mid \hat{\theta}_{i}^{\mathrm{ML}}\right)$.

Density Estimation. Some JDE problems are solved by density estimation. This is beyond the scope of this paper, which is for point inference.

\section{AN INTEGRATED APPROACH TO JDE}

\section{A. Benefit of Joint Solutions to JDE Problems}

Consider a JDE problem in which $x$ is the estimatee (i.e., the quantity to be estimated) and the decision involves $M$ candidates: $D_{1}, \ldots, D_{M}$. The complete Bayes solution to the problem of estimating $x$ using data $z$ is its posterior density $f(x \mid z)$. By the same token, a complete Bayesian solution to the problem of deciding on candidates $D_{1}, \ldots, D_{M}$ is the set of posterior probabilities $\left\{P\left\{D_{1} \mid z\right\}, \ldots, P\left\{D_{M} \mid z\right\}\right\}$. We call this set of probabilities soft decisions, although this is unorthodox in decision theory. In the same spirit, when $x$ and $D$ are not independent, we may be interested in inferring them jointly in terms of the set of functions $\left\{P\left[(D, x)_{1} \mid z\right], \ldots, P\left[(D, x)_{M} \mid z\right]\right\}$, where $P\left[(D, x)_{i} \mid z\right]$ is the posterior mixed "probability-density." Here the hybrid quantity $(D, x)_{i}=\left(x\left(D_{i}\right), D_{i}(x)\right)$ signifies the inter-dependence of $x$ and $D$.

If $x$ is not $D$-dependent, then $P\left[(D, x)_{i} \mid z\right]=$ $P\left\{D_{i}(x) \mid x, z\right\} f(x \mid z)$, which may be the case when $x$ is an unknown parameter common to all models $D_{i}$, as in classification of a signal where the hypotheses have a common unknown parameter. This suggests an estimationthen-decision method. If $D_{i}$ does not depend on $x$, then $P\left[(D, x)_{i} \mid z\right]=f\left(x \mid z, D_{i}\right) P\left\{D_{i} \mid z\right\}$. An example of this case is when $D_{i}$ is the $i$ th model of a system with the state $x$. This justifies a decision-then-estimation strategy.

The general case in which different $D$ may involve different $x$ has numerous examples. For target inference, this includes fusion of tracks $\left(x_{i}\right)$ that may correspond to different targets $\left(D_{i}\right)$, joint tracking $\left(x_{i}\right)$ and classification $\left(D_{i}\right)$ of targets, and tracking $\left(x_{i}\right)$ an unknown number $\left(D_{i}\right)$ of targets. In these cases, although we have $P\left[(D, x)_{i} \mid z\right]=$ $f\left(x_{i} \mid z, D_{i}\right) P\left\{D_{i}\left(x_{i}\right) \mid z\right\}=P\left\{D_{i} \mid x_{i}, z\right\} f\left(x_{i}\left(D_{i}\right) \mid z, D_{i}\right)$, the factorization does not really help because of the coupling. In the general case, therefore, an integrated approach would be more promising than separate decision and estimation as well as the traditional decision-then-estimation or estimation-thendecision approach. Note that a major challenge arises from the fact that while $x$ may be in a metric vector space, the decision space is neither metric nor a vector space. Another major difficulty is that $x$ or its density under different $D_{i}$ may be incommensurable.

Consider a simple problem of finding the peak location of the joint probability mass-density function $p(i, x)$ of a binarycontinuous pair of random variables $(I, X)$. Let $p_{i}=P\{I=$ $i\}=\int_{-\infty}^{\infty} p(i, x) d x$ be the probability point mass of $I$ and $f_{j}(x)=f(x \mid I=j)=\left.p(i, x)\right|_{i=j}$ the probability density function of $X$ given $\{I=j\}$, where $i=0,1$ and $-\infty<x<$ 
$\infty$. The joint decision and estimation will yield the location of the larger of $\max _{-\infty<x<\infty} f_{0}(x)$ and $\max _{-\infty<x<\infty} f_{1}(x)$, that is, $\arg \max _{i=0,1,-\infty<x<\infty} f_{i}(x)$, which is indeed the peak location of $p(i, x)$. However, the decision-then-estimation strategy amounts to the following: first select $j($ say, 1$)$ if $p_{1}$ is larger than $p_{0}$ and then find the peak location of $f_{1}(x)$; that is, it yields $\arg \max _{-\infty<x<\infty} f_{j}(x)$ with $j=\arg \max _{i=0,1} p_{i}$. This can be far from the peak location of $p(i, x)$ provided that the peak locations of $f_{0}(x)$ and $f_{1}(x)$ are quite different. This example demonstrates that for such problems it is better to solve the decision and estimation problems jointly in one shot, since the optimal joint solution is the globally optimal solution of the joint problem, while the solutions using the traditional two-stage strategy can be far from the globally optimal solution.

Due to practical considerations, we will focus on "point" JDE solutions in this paper.

\section{B. Bayes Decision and Bayes Estimation}

Our integrated approach to JDE is highly related to Bayes decision and Bayes estimation, described next.

Bayes Decision. In the Bayesian approach, the optimal decider is the one that minimizes the following Bayes risk

$$
\bar{R}_{D}=\sum_{i, j=1}^{N} c_{i j} P\left\{“ H_{i} ” \mid H_{j}\right\} P\left\{H_{j}\right\}
$$

where $P\left\{H_{j}\right\}$ is the prior probability of $H_{j}$ and $c_{i j}$ is the cost of " $H_{i}$ " (i.e., deciding on hypothesis $H_{i}$ ) while $H_{j}$ is true. When $i \neq j$, it is the cost of an incorrect decision, while $c_{i i}$ is the cost of a correct decision on $H_{i}$. The optimal Bayes decision decides on $H_{i}$ if its posterior cost $C_{i}(z)=$ $\sum_{j} c_{i j} P\left\{H_{j} \mid z\right\}$ is the smallest: $C_{i}(z) \leq C_{k}(z), \forall k$.

Bayes Estimation. An optimal Bayes estimator is a function of observations $z$ that minimizes the Bayes risk, $\bar{R}_{E}=$ $E[C(\tilde{x})]$, that is, $\hat{x}=\arg \min _{\hat{x}(z)} E[C(\tilde{x})]$, where $\bar{R}_{E}$ is the expectation of a cost function $C(\tilde{x})$ of the estimation error $\tilde{x}=x-\hat{x}$. Usually, the cost function should satisfy a set of admissibility conditions, including being symmetric about the origin, positive (semi)definite, and nondecreasing, as well as convex. The most widely used Bayes risk for estimation is $\bar{R}_{E}=E\left[\tilde{x}^{\prime} \tilde{x}\right]$, known as mean-square error. It is well known that the posterior mean, $\hat{x}=E[x \mid z]$, is the optimal estimator in this case (and in many other cases, including cases where the posterior density $f(x \mid z)$ is symmetric about its mean and $C(\tilde{x})$ is symmetric and convex).

\section{An Integrated Approach to JDE}

Novel Criterion for JDE. The cornerstone of our integrated approach to JDE is the introduction of the following Bayes risk for JDE

$$
\bar{R}=\sum_{i=1}^{M} \sum_{j=1}^{N}\left(\alpha_{i j} c_{i j}+\beta_{i j} E\left[C(x, \hat{x}) \mid D_{i}, H_{j}\right]\right) P\left\{D_{i}, H_{j}\right\}
$$

where $D_{i}$ stands for the $i$ th decision, which is equivalent to the event $\left\{z \in \mathcal{D}_{i}\right\}\left(\mathcal{D}_{i}\right.$ is the decision region for $D_{i}$ in the data space) without considering randomized decisions; $c_{i j}$ is the cost of decision $D_{i}$ when hypothesis $H_{j}$ is true; $C(x, \hat{x})$ is the estimation cost function; $E\left[C(x, \hat{x}) \mid D_{i}, H_{j}\right]$ is the expected cost conditioned on the fact that $D_{i}$ is decided but $H_{j}$ is true; $\alpha_{i j}$ and $\beta_{i j}$ are nonnegative relative weights of decision and estimation costs, which allows a variety of special cases.

This new Bayes risk $\bar{R}$ is a generalization of the traditional Bayes risk for decision, $\bar{R}_{D}$ of (1), and the traditional Bayes risk for estimation, $\bar{R}_{E}=E[C(\tilde{x})]$, respectively.

First and perhaps most important, the current theory of hypothesis testing has a very limited goal: to reject all hypotheses except one, say, $H_{i}$, and this is represented by the decision $D_{i}=$ " $H_{i}$ ". In other words, the decisions are confined to be in the form of $D_{i}=$ " $H_{i}$ " in the theory of hypothesis testing. In fact, the set of hypotheses $\left\{H_{1}, \ldots, H_{N}\right\}$ is our propositions concerning certain truth (simply put, they are a set of possible truth) and our decisions, albeit related to the hypotheses, do not have to be a judgment of the hypotheses directly. For example, people living in New Orleans devastated by Hurricane Katrina may have two hypotheses $-H_{1}$ : a major hurricane will hit New Orleans next year, and $H_{2}$ : this will not happen — but the decision candidates might be: $D_{1}$ : move out of New Orleans, $D_{2}$ : stay in New Orleans and move to a higher place, $D_{3}$ : lift the current house to a higher ground, $D_{4}$ : stay in New Orleans and do nothing to prepare for the hurricane. Our framework (2) allows such flexibility by not requiring a one-to-one correspondence between the set of decisions $\left\{D_{1}, \ldots, D_{M}\right\}$ and the set of hypotheses $\left\{H_{1}, \ldots, H_{N}\right\}$. In other words, $D_{i} \neq$ " $H_{i}$ " in general in our new formulation. As such, the decision part of this framework is already profoundly more general than hypothesis testing, which is limited to $D_{i}=$ " $H_{i}$ ". Several examples in Sec. IV demonstrate how powerful this generalization is. Amazingly, this generalization does not make the optimal solution any more complex.

Second, minimizing $\sum_{i, j} \beta_{i j} E\left[C(x, \hat{x}) \mid D_{i}, H_{j}\right] P\left\{D_{i}, H_{j}\right\}$ instead of $E[C(\tilde{x})]=\sum_{i, j} E\left[C(\tilde{x}) \mid D_{i}, H_{j}\right] P\left\{D_{i}, H_{j}\right\}$, the estimation part of this framework is also significantly more general than the traditional Bayes estimation: The cost function $C(x, \hat{x})$ generalizes $C(\tilde{x})$ to the case where $x$ and $\hat{x}$ may have different dimensions and the additional factors $\beta_{i j}$ convert possibly incommensurable conditional estimation risks $E\left[C(x, \hat{x}) \mid D_{i}, H_{j}\right]$ to a unified cost (money) so that the sum is sensible. For example, if $H_{i}$ stands for the truth that there are $i$ targets, then under $H_{1}$ and $H_{2}$, respectively, target state $x$ have different dimensions, but the estimator $\hat{x}$ must be the same for both cases since it does not know the truth, although its dimension could depend on the decision $D_{i}$ that there are $i$ targets. As a result, estimation error $\tilde{x}=x-\hat{x}$ and thus $E\left[C(\tilde{x}) \mid D_{i}, H_{j}\right]$ cannot be defined for both $H_{1}$ and $H_{2}$ in a unified way directly: what is the error of an estimator $\left(\hat{x}_{1}, \hat{x}_{2}\right)$ assuming two targets, each with a state dimension of $n$, while there is only a single target $x$ with a state dimension $n$ ? However, $E\left[C(x, \hat{x}) \mid D_{i}, H_{j}\right], \forall i, j$ can certainly be defined and $\beta_{i j}$ converts them all to cost. For instance, $\beta_{i 1} E\left[C(x, \hat{x}) \mid D_{i}, H_{1}\right]$ can be defined as the average cost (through factor $\beta_{i 1}$ ) $\sum_{k=1}^{i} E\left[C\left(x-\hat{x}_{k}\right)\right] P\left\{T_{k} \mid T \in\right.$ 
$\mathcal{T}\}$ or simply $E\left[C\left(x-\hat{x}_{k}\right)\right] / P\left\{T_{k} \mid T \in \mathcal{T}\right\}$ with $k=$ $\arg \min _{k=1, \ldots, i} E\left[C\left(x-\hat{x}_{k}\right)\right]$, where $T_{k}=\left\{\hat{x}_{k}\right.$ is the estimate of the state $x$ of the true target $T\}, T_{0}=$ \{none of $\hat{x}_{k}$ is the state estimate of the true target $\left.T\right\}$, and $\mathcal{T}=$ $\left\{T_{0}, T_{1}, \ldots, T_{k}\right\}$. (Note that there is no flawless Bayes risk for all applications.) This generality enables our formulation to handle effectively a much larger class of problems involving conditional subproblems that are incommensurable. This is needed for many applications, including in particular tracking an unknown number of targets, considered in Sec. IV.

Third, if $M=N$ and $D_{i}=$ " $H_{i}$ " for all $i$, then: (a) our joint risk $\bar{R}$ with $\left(\alpha_{i j}, \beta_{i j}\right)=(1,0)$ and $(0,1)$ reduces to the traditional Bayes risk for decision and estimation, respectively; (b) $\bar{R}$ is simply the sum of the traditional Bayes risks for decision and estimation respectively if $\left(\alpha_{i j}, \beta_{i j}\right)=(1,1)$; (c) $\bar{R}$ is a weighted sum of the product of decision and estimation costs if $\left(\alpha_{i j}, \beta_{i j}\right)=\left(0, c_{i j}\right)$. By an appropriate choice of $\alpha_{i j}$ and $\beta_{i j}$, our new framework is suitable for all three classes of JDE problems: (a) decision and estimation are virtually equally important, (b) decision is primary and estimation is secondary (e.g., testing hypotheses with unknown parameters), and (c) estimation is primary and decision is secondary (e.g., estimation with several possible true models). In other words, the relative weight of decision and estimation in a JDE problem can be captured by the relative magnitudes of $\alpha_{i j}$ and $\beta_{i j}$.

In short, this new Bayes risk generalizes the traditional ones in that (a) it is a risk for joint decision and estimation, (b) the decision set and the hypothesis set need not be one-to-one correspondent, (c) it can handle problems with incommensurable subproblems of estimation, and (d) the coefficients $\left(\alpha_{i j}, \beta_{i j}\right)$ provide additional flexibilities.

Determination of $\alpha_{i j}$ and $\beta_{i j}$ is a design issue and a topic for future research. Clearly, $\alpha_{i j} c_{i j}$ for $i \neq j$ should be larger than $\alpha_{i i} c_{i i}$ in general to penalize incorrect decisions. Contrary to many people's intuition, $\beta_{i j}$ for $i \neq j$ should be smaller than $\beta_{i i}$ in general so that the JDE solution is sensitive to estimation performance. More comments are given in the context of a track-fusion example in Sec. IV and some results for joint tracking and classification are given in [20].

Optimal JDE solution. This formulation provides a basis for an integrated approach to JDE. Effective Bayes JDE solutions can be developed in this framework. While various cost functions $C(x, \hat{x})$ are possible, our presentations below are for the case with $C(x, \hat{x})=\tilde{x}^{\prime} \tilde{x}$, which have widespread applications, particularly for dynamic systems.

Theorem 1. To minimize $\bar{R}$ of (2), for any given $E\left[C(x, \hat{x}) \mid D_{i}, H_{j}\right]$, the optimal decision $D$ is

$$
D=D_{i} \quad \text { if } \quad C_{i}(z) \leq C_{k}(z), \forall k
$$

where the posterior cost is given by

$$
C_{i}(z)=\sum_{j}\left(\alpha_{i j} c_{i j}+\beta_{i j} E\left[C(x, \hat{x}) \mid D_{i}, H_{j}\right]\right) P\left\{H_{j} \mid z\right\}
$$

and given any set of regions $\left\{\mathcal{D}_{1}, \ldots, \mathcal{D}_{M}\right\}$ (not necessarily a partition) of the data space, the optimal estimator for (2) with
$C(x, \hat{x})=\tilde{x}^{\prime} \tilde{x}$ is the following generalized posterior mean

$$
\hat{x}=\sum_{i, j} \hat{x}_{i j} \bar{P}\left\{D_{i}, H_{j} \mid z\right\}
$$

where, for $z \in \mathcal{D}_{i}$,

$$
\begin{aligned}
\hat{x}_{i j} & =E\left[x \mid z, D_{i}, H_{j}\right]=\hat{x}_{j}=E\left[x \mid z, H_{j}\right] \\
\bar{P}\left\{D_{i}, H_{j} \mid z\right\} & =\frac{\beta_{i j} P\left\{D_{i}, H_{j} \mid z\right\}}{\sum_{l, k} \beta_{l k} P\left\{D_{l}, H_{k} \mid z\right\}} \\
& =\frac{\beta_{i j} P\left\{H_{j} \mid z\right\}}{\sum_{l, k} 1\left(z ; \mathcal{D}_{l}\right) \beta_{l k} P\left\{H_{k} \mid z\right\}} \\
1\left(z ; \mathcal{D}_{i}\right) & = \begin{cases}1, & z \in \mathcal{D}_{i} \\
0, & \text { else }\end{cases} \\
P\left\{H_{j} \mid z, D_{i}\right\} & =P\left\{H_{j} \mid z\right\}
\end{aligned}
$$

and these quantities are not defined if $z \notin \mathcal{D}_{i}$. If $z \in \mathcal{D}_{i}$ implies $z \notin \mathcal{D}_{k}, \forall k \neq i$ (e.g., when $\left\{\mathcal{D}_{1}, \ldots, \mathcal{D}_{M}\right\}$ forms a partition of the data space), then (5) is simplified to the following generalized posterior mean

$$
\hat{x}=\sum_{i} 1\left(z ; \mathcal{D}_{i}\right) \check{x}_{i}
$$

where, for $z \in \mathcal{D}_{i}$,

$$
\begin{aligned}
\check{x}_{i} & =\bar{E}[x \mid z]=\sum_{j} \hat{x}_{j} \bar{P}\left\{H_{j} \mid z\right\} \\
\bar{P}\left\{H_{j} \mid z\right\} & =\frac{\beta_{i j} P\left\{H_{j} \mid z\right\}}{\sum_{k} \beta_{i k} P\left\{H_{k} \mid z\right\}}
\end{aligned}
$$

and they are undefined if $z \notin \mathcal{D}_{i}$.

Proof. Omitted, due to space limitation.

Remarks. Let $D_{i}=$ " $H_{i}$ ". Then the above optimal decision reduces to the traditional optimal decision if $\alpha_{i j}$ and $\beta_{i j}$ are such that $\alpha_{i j} c_{i j}+\beta_{i j} E\left[C(x, \hat{x}) \mid D_{i}, H_{j}\right]=c_{i j}$ (e.g., $\left.\left(\alpha_{i j}, \beta_{i j}\right)=(1,0)\right)$. It is thus clear that the quality/accuracy of the associated estimation is accounted for in the above optimal decision, but not in the traditional optimal decision. Also, our generalization of decisions to $D_{i} \neq$ " $H_{i}$ " incurs no extra difficulty (e.g., (3)-(4) remains unchanged whether $D_{i}=$ " $H_{i}$ " or not). Under the idealized assumption that there is no decision error-namely, $P\left\{H_{j} \mid D_{i}, z\right\}=\delta_{i-j}$ if $z \in \mathcal{D}_{i}$ and undefined otherwise, where $\delta_{i-j}$ is the Kronecker delta: $\delta_{i-j}=1$ if $i=j$ and 0 otherwise-the above optimal estimator reduces to the estimator in the traditional decisionthen-estimation procedure:

$$
\hat{x}=\sum_{i} 1\left(z ; \mathcal{D}_{i}\right) E\left[x \mid z, H_{i}\right]
$$

Note the similarity and difference between (6) and (7): If the decision is $D_{i}$, the corresponding estimator (7) is the conditional mean $\hat{x}_{i}=E\left[x \mid z, H_{i}\right]$, but the optimal estimator (6) is $\check{x}_{i}=\bar{E}[x \mid z]$, a reweighted probabilistic weighted sum of the conditional means to account for possible decision errors, where the weights depend on $i$. This comparison makes several serious drawbacks of the traditional approach explicit and quantifiable. For example, possible decision errors are not accounted for in estimation; decision is made without 
taking into account its impact on the quality of the estimation (e.g., even if such a decision leads to poor estimation). This comparison also reveals part of the reason why a good soft decision based estimator outperforms a good hard decision based one for many JDE problems, as evidenced by the success of multiple-model estimation algorithms [18]. Alternatively, the above optimal estimator with $\beta_{i j}=\delta_{i-j}$ also reduces to the estimator in the traditional decision-then-estimation procedure, as can be seen by $\check{x}_{i}=\hat{x}_{i}$ in this case.

If the conditional mean $\hat{x}_{j}=E\left[x \mid z, H_{j}\right]$ needed for the above optimal estimator is not available, it may be replaced by the corresponding linear minimum mean-square error (LMMSE) estimator, resulting in a simple nonlinear estimator as a data-dependent weighted sum of LMMSE estimators. It is easily implementable and outperforms the single LMMSE estimator, as evidenced by the superior performance of the Interacting Multiple-Model (IMM) estimator to the LMMSE estimator [9], [18], since the IMM estimator is also a datadependent weighted sum of LMMSE estimators.

Sometimes a JDE problem is handled by ignoring the coupling and solving the decision and estimation problems separately. In such a case, it is possible for a JDE procedure to beat the "optimal" estimator and the "optimal" decider simultaneously. The key lies in the type of available data. Suppose we have an imager and a radar. The radar provides kinematic data that are valuable for target state estimation but have little value for decision (say, target identification) directly (i.e., without going through decision); the information from the imager is invaluable for target identification, but has little value for target state estimation directly (i.e., without going through estimation) due to, say, poor image registration. The normal (and approximate) implementations of the optimal decider (target identifier) and the optimal state estimator would rely entirely on the imagery and the kinematic data, respectively. A JDE procedure utilizes both the imagery and the kinematic measurements. If target identity and motion are coupled, as is often the case, through the JDE procedure that takes advantage of the inter-dependence between the target motion and the target identity, the imagery will help target state estimation significantly and the kinematic data will facilitate target identification considerably. Therefore, the optimal JDE procedure may beat (the approximate but usual implementation of) the optimal estimator and the optimal decider simultaneously. This is illustrated in [20].

\section{A JDE Algorithm}

Since the optimal JDE solution is the joint of (3) and (6), we have the following JDE algorithm:

- Initialization: Give an initial partition $\mathcal{D}^{(0)}=$ $\left\{\mathcal{D}_{1}^{(0)}, \ldots, \mathcal{D}_{M}^{(0)}\right\}$ of the data space.

- E-Step: For the given partition $\mathcal{D}^{(k)}=\left\{\mathcal{D}_{1}^{(k)}, \ldots, \mathcal{D}_{M}^{(k)}\right\}$ of the data space, compute the optimal estimator $\hat{x}^{(k)}$ given by (6).

- D-Step: Compute the conditional mse $\mathcal{E}_{i j}^{(k)}=$ $E\left[\tilde{x}^{(k) \prime} \tilde{x}^{(k)} \mid D_{i}^{(k)}, H_{j}\right], \forall i, j, \quad$ and $\quad C_{i}^{(k+1)}(z)=$ $\sum_{j}\left(\alpha_{i j} c_{i j}+\beta_{i j} \mathcal{E}_{i j}^{(k)}\right) P\left\{H_{j} \mid z\right\}, \forall i, \quad$ where $\tilde{x}^{(k)}=x-\hat{x}^{(k)}$. Then the optimal partition is given by $\mathcal{D}^{(k+1)}=\left\{\mathcal{D}_{1}^{(k+1)}, \ldots, \mathcal{D}_{M}^{(k+1)}\right\}$, where $\mathcal{D}_{i}^{(k+1)}=\left\{z: C_{i}^{(k+1)}(z) \leq C_{m}^{(k+1)}(z), \forall m\right\}$.

- Repeat the E-step and D-step until the relative reduction in the Bayes risk $\bar{R}$ of (2) in one iteration is smaller than a threshold.

Alternatively, it is equally good to start with an initial estimator $\hat{x}^{(0)}$ and proceed with a D-step.

Theorem 2. The above JDE algorithm converges.

Proof. Omitted, due to space limitation.

Remarks. If $z \in\left(\mathcal{D}_{i}^{(k)} \cap \mathcal{D}_{i}^{(k+1)}\right)$, then $\bar{P}\left\{H_{j} \mid z, D_{i}^{(k)}\right\}=$ $\bar{P}\left\{H_{j} \mid z, D_{i}^{(k+1)}\right\}, \check{x}_{i}^{(k)}=\check{x}_{i}^{(k+1)}$, and $\hat{x}^{(k)}=\hat{x}^{(k+1)}$. However, this does not imply $\mathcal{E}_{i j}^{(k)}=\mathcal{E}_{i j}^{(k+1)}, \forall i, j$, because

$\mathcal{E}_{i j}^{(k)}=E\left[\tilde{x}^{(k) \prime} \tilde{x}^{(k)} \mid D_{i}^{(k)}, H_{j}\right]=\int_{z \in \mathcal{D}_{i}^{(k)}} \tilde{x}^{(k) \prime} \tilde{x}^{(k)} d F\left(x, z \mid H_{j}\right)$

(The integration region changes although the integrand does not.) As such, the E-step is needed in the iteration if and only if the D-step leads to a different decision (not partition) than in the previous iteration. It is reasonable to expect that for most cases, the D-step will not lead to a different decision, especially when $z$ is not close to the boundary of the decision region. So, in practice, the iteration can be terminated if $z$ is not close to the boundary. This has the following important implication for the application of this JDE algorithm: Although it may take many iterations for the algorithm to settle down at the optimal JDE rule $(D(\cdot), \hat{x}(\cdot))$, it usually takes only a few iterations to settle down at the optimal joint decision and estimate $(D(z), \hat{x}(z))$ for a given $z$. This is because to make an optimal joint decision and estimate for given data is usually much easier than to find the optimal JDE rule. More specifically, assume that we find $\mathcal{D}^{(0)}$ by the optimal Bayes decision rule for $\bar{R}_{1}=\sum_{i=1}^{M} \sum_{j=1}^{N} \alpha_{i j} c_{i j} P\left\{D_{i}, H_{j}\right\}$, that is, $\mathcal{D}^{(0)}=\left\{\mathcal{D}_{1}^{(0)}, \ldots, \mathcal{D}_{M}^{(0)}\right\}$, where $\mathcal{D}_{i}^{(0)}=\left\{z: C_{i}^{(0)}(z) \leq\right.$ $\left.C_{m}^{(0)}(z), \forall m\right\}$ with $C_{m}^{(0)}(z)=\sum_{j} \alpha_{m j} c_{m j} P\left\{H_{j} \mid z\right\}$. Then, for most cases (i.e., when the data lies in a place not close to the boundary of the optimal decision region) $z \in\left(\mathcal{D}_{i}^{(k)} \cap\right.$ $\mathcal{D}_{i}^{(k+1)}$ ), where $k=1$ or 2 , and thus the iteration stops at $k+1$. Only for the cases where the data lies in a place close to the boundary of the decision region, the iteration may go on. It is the existence of these data points that entails additional iterations in finding the optimal JDE rule.

The above stopping criterion requires the computation of Bayes risk $\bar{R}$, which is not so simple (e.g., $P\left\{D_{i} \mid H_{j}\right\} P\left\{H_{j}\right\}, \forall i, j$, are needed). Alternatively, we may stop the iteration if there is no change in the decision (i.e., $\left.z \in\left(\mathcal{D}_{i}^{(k)} \cap \mathcal{D}_{i}^{(k+1)}\right)\right)$ and $\max _{i, j}\left|\mathcal{E}_{i j}^{(k)}-\mathcal{E}_{i j}^{(k+1)}\right| / \mathcal{E}_{i j}^{(k)}$ is below a threshold.

\section{ILlustrative Applications in TARget Inference}

Track fusion. As a focal point of data fusion research for decades, track fusion-fusion of tracks from multiple sensors to improve track accuracy and quality-is much more challenging than distributed filtering or estimation fusion because 
it involves uncertainties in measurement origin, target class, target motion model, the number of targets, etc. as well as noise. Fusion of tracks from multiple sensors has been divided into two steps so far [2], following the traditional decisionthen-estimation approach. The first step, track-to-track association, decides whether tracks from two or more sensors are for the same target. This is followed by an estimation step, track-to-track fusion, which combines the tracks from multiple sensors deemed for the same target by estimation fusion rules. This two-step approach has been taken for granted but it suffers from serious drawbacks. For example, possible decision errors in track-to-track association are ignored in track-to-track fusion so far. Also, the knowledge about the accuracy of each fused track is usually not used in the track-to-track association. Clearly, track fusion is a JDE problem and is better solved by an integrated approach, rather than the existing two-step approach. Our proposed integrated approach above can be applied to track fusion naturally, resulting in integrated track fusion or joint track association and fusion. It does not have the drawbacks mentioned above. To our knowledge, such an integrated approach has never been proposed.

Consider a two-sensor case with possibly two targets (a tank and a truck, see Fig. 1) where sensor 1 has a track of the truck and a false track (i.e., one that is not related to either target) and sensor 2 has a track of each target plus a false track, but the truth is not known to the track fuser. One implementation of our integrated approach for this example with five tracks (two tracks $\hat{x}^{1}$ from sensor 1 and three tracks $\hat{x}^{2}$ from sensor 2 ) is as follows. Denote tank, truck, and false track by $A, B$, and $F$, respectively. For each track pair $\left(\hat{x}^{1}, \hat{x}^{2}\right)$ from the two sensors, take the ordered pair as the measurement, namely, $z=\left(\hat{x}^{1}, \hat{x}^{2}\right)$ (or $z=\left[\left(\hat{x}^{1}, \hat{x}^{2}\right),\left(d_{1}, d_{2}\right)\right]$ if the corresponding decisions $\left(d_{1}, d_{2}\right)$ for the target class are known), and let the set of hypotheses and the set of decisions be

$$
\begin{gathered}
\left(H_{1}, H_{2}, H_{3}^{\prime}, \ldots, H_{9}^{\prime}\right) \\
=((A, A),(B, B),(A, B),(A, F),(B, A) \\
(B, F),(F, A),(F, B),(F, F)) \\
\left(D_{1}, D_{2}, D_{3}\right)=((A, A),(B, B),\{\text { all other ordered pairs }\})
\end{gathered}
$$

where, e.g., $(A, A)$ stands for a tank-tank track pair. Note that $(A, A)$ and $(B, B)$ may be different not only by their target class but also by their dynamics. The problem of minimizing (2) with $C(x, \hat{x})=\tilde{x}^{\prime} \tilde{x}$ can be solved, as given by (3) and (6). If the solution is, say, $(D, \hat{x})=\left(D_{1}, \hat{x}_{1}\right)$, then we have a fused (improved) track $\hat{x}_{1}$ deemed for the tank, given by

$$
\begin{aligned}
\hat{x}_{1} & =\frac{E[x \mid z,(A, A)] \beta_{11} P\{(A, A) \mid z\}}{\beta_{11} P\{(A, A) \mid z\}+\beta_{22} P\{(B, B) \mid z\}} \\
& +\frac{E[x \mid z,(B, B)] \beta_{22} P\{(B, B) \mid z\}}{\beta_{11} P\{(A, A) \mid z\}+\beta_{22} P\{(B, B) \mid z\}}
\end{aligned}
$$

where $E[x \mid z,(A, A)]$ is obtained by estimation fusion rules assuming both tracks are for the tank (likewise for $E[x \mid z,(B, B)])$ and $P\{(A, A) \mid z\}$ is the corresponding posterior probability. Note that $\hat{x}_{1}$ is deemed the estimate of the tank's state, but it accounts for the possibility that it is actually a truck. The track pair should not be fused if $D=D_{3}$. We may choose large $\alpha_{3 j} c_{3 j}$ for $j=1,2$ and large $\alpha_{i j} c_{i j}$ for $i=1,2$ and $j \geq 3$ in (2) to penalize false decisions so that they occur rarely. We may also choose large $\beta_{i i}$ for $j=1,2$ so that the criterion (2) is sensitive to estimation error. Note that our new Bayes formulation allows the choice of decisions $\left\{D_{1}, D_{2}, D_{3}\right\}$, which is more reasonable than what the traditional formulation $D_{i}=$ " $H_{i}$ " would lead to:

$$
\begin{aligned}
&\left(D_{1}, D_{2}, D_{3}^{\prime}, \ldots, D_{9}^{\prime}\right) \\
&=((A, A),(B, B)(A, B),(A, F),(B, A) \\
&(B, F),(F, A),(F, B),(F, F))
\end{aligned}
$$

For instance, $D_{2}$ may beat each of $D_{3}^{\prime}, \ldots, D_{9}^{\prime}$ individually, but not $D_{3}$ and thus using $\left\{D_{1}, D_{2}, D_{3}^{\prime}, \ldots, D_{9}^{\prime}\right\}$ would lead to a decision $D_{2}$ whereas using $\left\{D_{1}, D_{2}, D_{3}\right\}$ would lead to $D_{3}$. Of course, we may also choose the alternative set of hypotheses

$$
\left(H_{1}, H_{2}, H_{3}\right)=((A, A),(B, B),\{\text { all other ordered pairs }\})
$$

so that the traditional decision framework works for $\left\{D_{1}, D_{2}, D_{3}\right\}=\left\{\right.$ " $H_{1} "$ " " $H_{2} "$ " " $H_{3}$ " $\}$. As can be seen from $C_{i}(z)$ of (3), however, this choice leads to, for $i=1,2$,

$$
C_{i}(z)=\sum_{k=1,2,3}\left(\alpha_{i k} c_{i k}+\beta_{i k} E\left[C(x, \hat{x}) \mid D_{i}, H_{k}\right]\right) P\left\{H_{k} \mid z\right\}
$$

which differs from the one with our choice $\left\{H_{1}, H_{2}\right.$, $\left.H_{3}^{\prime}, \ldots, H_{9}^{\prime}\right\}$ and $\left\{D_{1}, D_{2}, D_{3}\right\}$, given by, for $i=1,2$,

$$
C_{i}(z)=\sum_{j=1,2,3, \ldots, 9}\left(\alpha_{i j} c_{i j}+\beta_{i j} E\left[C(x, \hat{x}) \mid D_{i}, H_{j}\right]\right) P\left\{H_{j} \mid z\right\}
$$

Clearly, our choice with $\left\{H_{1}, H_{2}, H_{3}^{\prime}, \ldots, H_{9}^{\prime}\right\}$ and $\left\{D_{1}, D_{2}, D_{3}\right\}$ is more flexible to express different cases in $\left\{H_{3}^{\prime}, \ldots, H_{9}^{\prime}\right\}$-it allows detailed treatment of each case in $\left\{H_{3}^{\prime}, \ldots, H_{9}^{\prime}\right\}$, while the choice $\left\{H_{1}, H_{2}, H_{3}\right\}$ lumps all the cases in $\left\{H_{3}^{\prime}, \ldots, H_{9}^{\prime}\right\}$ into a single $H_{3}$.

This example demonstrates two important points: (a) the generalization that $D_{i}$ need not be " $H_{i}$ " is very significant and (b) track-to-track association and fusion can be done jointly and optimally.

Clearly, our approach works for track fusion regardless whether each track from a sensor has an estimated target ID (or decision) or not. If it does, the problem is actually one of fusing tracks and target IDs (or decisions) jointly, or JDE fusion. This manifests that our new Bayes paradigm enables an integrated approach to JDE fusion. The approach is integrated in that the continuous-valued tracks and discrete-valued decisions are handled jointly. Besides fusion of tracks and decisions, it can also fuse tracks with non-kinematic attributes or features, extracted from, e.g., imaging sensors. The attributes may include track quality and discriminative feature information. Useful discriminative features can be target ID, target type, target signatures, or target signal strength, etc. The quality measure of a track quantifies how likely the track is really a manifestation of a target. It is useful for determining whether tracks from different sensors are for the same target. One of 
the most promising directions in target inference is to take advantage of the feature information obtained from multiple sensors. Feature-aided tracking and recognition is in essence fusion of feature information and kinematic data. Not only should fused tracks and decisions/attributes/features pair up, but fusion of tracks and fusion of decisions/attributes/features are also better done jointly.

Joint detection and tracking. Our JDE framework also provides a basis for an integrated approach to detection and tracking of an unknown number of targets in an uncertain environment. Suppose we know that at most two targets are present in the "gate"-a local region that has a large probability of capturing the target-originated measurements if a target is present. Given, say, three measurements $z_{1}, z_{2}, z_{3}$ in the gate at a time, each could be false alarms or have originated from any or both of the targets due to clutter and limited sensor resolution, and each target may generate zero, one, or more contacts because of imperfect detection, multipaths, etc. We may cast this difficult problem into the above JDE framework with hypotheses $\left\{H_{0}, H_{1}, H_{2}, H_{3}\right\}$ and decisions $\left\{D_{0}, D_{1}, D_{2}\right\}$, where $H_{j}=$ " $j$ measurements are target originated" and $D_{i}=$ "there are $i$ targets". Note again that the freedom from the required one-to-one correspondence between the decision set and the hypothesis set opens a lot of avenues to solving many difficult problems. The decision regions form a partition of the product space of the three measurements $z_{1}, z_{2}, z_{3}$. Suppose our JDE solution (6) with $C(x, \hat{x})=\tilde{x}^{\prime} \tilde{x}$ yields $(D, \hat{x})=\left(D_{1}, \hat{x}_{1}\right)$, meaning that the best inference is that one target is present $\left(D_{1}\right)$ with the state estimate

$\hat{x}_{1}=\sum_{j=0,1,2,3} E\left[x \mid z, H_{j}\right] \frac{\beta_{1 j} P\left\{H_{j} \mid z\right\}}{\sum_{j} \beta_{1 j} P\left\{H_{j} \mid z\right\}}, z=\left\{z_{1}, z_{2}, z_{3}\right\}$

which is a weighted sum of the conditional means assuming that $0,1,2$, and 3 measurements, respectively, were originated from the target since the decision is $D_{1}$. These conditional means can be obtained by existing tracking methods assuming one target is present and $j$ measurements are target originated, respectively. (If our JDE solution (6) yields $(D, \hat{x})=\left(D_{2}, \hat{x}_{2}\right)$, then $\hat{x}_{2}$ would be an estimate of the stacked vector of the state of the two targets.) Note that the accuracy $E\left[\tilde{x}^{\prime} \tilde{x} \mid D_{i}, H_{j}\right]$ of the decision-directed estimation is a part of the cost of decision $D_{i}$ under $H_{j}$ in our framework, but not in the traditional decision or the decision part of the traditional decision-thenestimation approach.

Tracking an unknown number of targets is very difficult. For example, a different number of targets have a different stacked state dimension and thus their conditional means are not summable. Although tremendous efforts have been made on multitarget tracking for decades, almost all existing methods either assume a known number of targets or determine the number first and then do tracking accordingly following the traditional decision-then-estimation approach. This example illustrates that our new JDE framework is capable of tracking an unknown number of targets in the presence of measurementorigin uncertainty in a coherent, systematic manner. Such a framework was not available before, except in a highly abstract random-set theoretic setting that will be beyond practitioners for a long time. Our formulation provides a simple and easily implementable framework for this de facto randomset problem by including $\sum_{i, j} \beta_{i j} E\left[\tilde{x}^{\prime} \tilde{x} \mid D_{i}, H_{j}\right] P\left\{D_{i}, H_{j}\right\}$ in the Bayes risk (2). Note that summing up $E\left[\tilde{x}^{\prime} \tilde{x} \mid D_{i}, H_{j}\right]$ for different $(i, j)$ pairs in general is not sensible without converting them all to cost (money) by $\beta_{i j}$.

Joint tracking and classification. Another natural application of our integrated approach is joint tracking and classification, which has received increasing attention in recent years. To illustrate, consider a simple example of tracking and classifying two moving targets, a tank and a truck, with different dynamics using such observations as images and kinematic measurements (see Fig. 1). Tracking and classification are really joint for unresolved targets, e.g., crossing targets where two or more tracks become inseparable for a while and then separable again afterwards. In the traditional decision-then-estimation approach, a target is classified first, say, as a tank, based on observations or extracted features and then tracked using measurements based on tank's dynamic model. A misclassification will lead to a usually much larger tracking error than a correct classification. For instance, two crossing targets (tank and truck) may be deemed moving straight without a turn during crossing while they actually both took a turn - the tank ID and truck ID are mistakenly swapped (see Fig. 1). Such misclassification may have a dire consequence - the truck will be destroyed if we intend to hit the tank. In this case, the actual tracking error-the difference between the actual, say, tank state and its estimate-would be extremely large. This error information is useful for an improved classification, but it is not so used in the traditional two-stage approach. It may be used to update the classification results in various ways. Such joint tracking and classification problems can be formulated as that of minimizing our joint Bayes risk (2). As such, an integrated, systematic solution can be obtained. More specifically, a target class along with the corresponding dynamic model can be defined as a hypothesis. If we choose $C(x, \hat{x})=\tilde{x}^{\prime} \tilde{x}$ and $D_{i}=$ " $H_{i}$ " in (2), then the optimal joint classifier-tracker is given by (3) and (6). The classifier and tracker work as a team optimally: the classifier takes account of the tracking error by favoring a classification that leads to a smaller tracking error, since the tracking error is part of the classification cost; the tracker considers the possibility of misclassification by using a weighted sum of estimates, each being a posterior mean conditioned on a target class, rather than relies entirely on the class chosen by the classifier. As such, an optimally determined class and track of the target is obtained. Note that this optimal tracker is in fact a generalized multiple class-motion model estimator. A conventional multiple-model (MM) estimator would not work well for such applications as state estimation of crossing targets since they ignore the coupling between target class and state, which would be devastating here. For other tracking applications, such a tracker will outperform the traditional tracker conditioned on the single class chosen by a classifier, 
as evidenced by the performance superiority of MM estimation to decision-based estimation [18]. The flexibility in choosing $\beta_{i j}$ empowers our formulation to handle a variety of applications effectively. A simple yet representative example of joint tracking and classification is given in [20] that compares our joint solution with other approaches, along with design issues.

Even if the target class is not considered, crossing targets (more generally, closely-spaced targets) present a challenge for tracking mainly due to the difficulty in solving the underlying data association problem. Numerous algorithms have been proposed in recent years. This is clearly a JDE problem, conceptually similar to the problem of track fusion discussed above. The above formulation of ours is also good for this problem and for many other problems in tracking, in particular, the "standard" data association problem arising from clutter and imperfect detection, which has been the core problem of target tracking and studied extensively for decades. An additional example is tracking in the presence of countermeasures, for which a systematic approach was proposed in [19], [25].

\section{SUMMARY}

A novel and integrated approach to joint decision and estimation has been proposed in a general setting that minimizes a new Bayes risk as a generalization of those for decision and estimation, respectively. It is theoretically superior to the existing approaches. Also presented are the structure of the optimal JDE and a converging JDE algorithm in this framework. The power of the proposed approach has been illustrated by its flexibility of formulating several important problems in target inference.

\section{REFERENCES}

[1] D. Angelova and L. Mihaylova. Sequential Monte Carlo Algorithms for Joint Target Tracking and Classification Using Kinematic Radar Information. In Proc. 2004 International Conf. on Information Fusion, volume II, pages 709-716, Stockholm, Sweden, June 28-July 12004.

[2] Y. Bar-Shalom and X. R. Li. Multitarget-Multisensor Tracking: Principles and Techniques. YBS Publishing, Storrs, CT, 1995.

[3] B. Baygun and A. O. Hero. Optimal Simultaneous Detection and Estimation Under a False Alarm Constraint. IEEE. Trans. Information Theory, IT-41(3):688-703, May 1995.

[4] R. E. Bethel. Joint Detection and Estimation in a Multiple Signal Array Processing Environment. $\mathrm{PhD}$ thesis, George Mason University, Fairfax, VA, USA, 2002.

[5] E. P. Blasch and C. Yang. Ten Ways to Fuse GMTI and HRRR Measurements for Joint Tracking and Identification. In Proc. 2004 International Conf. on Information Fusion, volume II, pages 1006-1013, Stockholm, Sweden, June 2004.

[6] Y. Boers and H. Driessen. Integrated Tracking and Classification: An Application of Hybrid State Estimation. In Proc. 2001 SPIE Conf. on Signal and Data Processing of Small Targets, vol. 4473, pages 198-209, 2001.

[7] S. Challa and G. W. Pulford. Joint Target Tracking and Classification Using Radar and ESM Sensors. IEEE Trans. Aerospace and Electronic Systems, 37(3):1039-1055, 2001.

[8] H. Chen, X. R. Li, and Y. Bar-Shalom. On Joint Track Initiation and Parameter Estimation under Measurement Origin Uncertainty. IEEE Trans. Aerospace and Electronic Systems, 40(2):675-694, Apr. 2004.

[9] O. L. V. Costa. Linear Minimum Mean Square Error Estimation for Discrete-Time Markovian Jump Linear Systems. IEEE Trans. Automatic Control, AC-39(8):1685-1689, Aug. 1994.

[10] E. Fishler and H. Messer. Detection and Parameter Estimation of a Transient Signal Using Order Statistics. IEEE Ttransactions on Signal Processing, 48(5):1455-1458, May 2000.
[11] N. Gordon, S. Maskell, and T. Kirubarajan. Efficient Particle Filters for Joint Tracking and Classification. In Proc. 2002 SPIE Conf. on Signal and Data Processing of Small Targets, vol. 4728, pages 439449, Orlando, FL, USA, April 2002.

[12] S. Herman and P. Moulin. A Particle Filtering Approach to FM-Band Passive Radar Tracking and Automatic Target Recognition. In Proc. IEEE Aerospace Conf., volume 4, pages 1789-1808, 2002.

[13] S.M. Herman. A Particle Filtering Approach to Joint Passive Radar Tracking and Target Classification. PhD thesis, Univ. of Illinois at Urbana-Champaign, 2002.

[14] D. Khosla and Y. Chen. Joint Kinematic and Feature Tracking Using Probabilistic Argumentation. In Proc. 2003 International Conf. on Information Fusion, pages 11-16, Cairns, Australia, July 2003.

[15] M. Krieg. Joint Multi-sensor Kinematic and Attribute Tracking Using Bayesian Belief Network. In Proc. 2003 International Conf. on Information Fusion, pages 17-24, Cairns, Australia, July 2003.

[16] A. D. Lanterman. Tracking and Recognition of Airborne Targets via Commercial Television and FM Radio Signals. In Proc. of SPIE Conf. on Acquisition, Tracking, and Pointing, XIII, volume 3692, pages 189198, Orlando, FL, 1999.

[17] Q. Li, C.N. Georghiades, and X. Wang. Joint Sequential Channel Estimation and Multiuser Detection for Uplink CDMA over Multipath Fading. In Proc. IEEE 56th Vehicular Technology Conf., volume 3, pages $1882-1886$, Sep. 2002.

[18] X. R. Li and V. P. Jilkov. Survey of Maneuvering Target Tracking. Part V: Multiple-Model Methods. IEEE Trans. Aerospace and Electronic Systems, (4):1255-1321, Oct. 2005.

[19] X. R. Li, B. J. Slocumb, and P. D. West. Tracking in the Presence of Range Deception ECM and Clutter by Decomposition and Fusion. In Proc. 1999 SPIE Conf. on Signal and Data Processing of Small Targets, vol. 3809, pages 198-210, Denver, CO, USA, July 1999.

[20] X. R. Li, M. Yang, and J.-F. Ru. Joint Tracking and Classification Based on Bayes Joint Decision and Estimation. In Proc. 2007 International Conf. on Information Fusion, Québec City, Canada, July 2007.

[21] W. Mei, G.-L. Shan, and X. R. Li. An Efficient Bayesian Algorithm for Joint Target Tracking and Classification. In Proc. 7th International Conf. on Signal Processing, Beijing, China, Aug. 31-Sept. 42004.

[22] M.I. Miller, A. Srivastava, and U. Grenander. Conditional-Mean Estimation Via Jump-Diffusion Process in Multiple Target Tracking/Recognition. IEEE Trans. Signal Processing, 43(11):2678-2690, 1995.

[23] J.A. O'Sullivan, S.P. Jacobs, M.I. Miller, and D.L. Synder. A LikelihoodBased Approach to Joint Target Tracking and Identification. In Proc. 27th Asilomar Conf. on Signals, Systems and Computers, pages 290294, Nov. 1993.

[24] J. A. O'Sullivan S. P. Jacobs. High Resolution Radar Models for Joint Target Tracking and Recognition. In IEEE International Radar Conference, Syracuse, NY, USA, May 1997.

[25] B. J. Slocumb, P. D. West, and X. R. Li. Implementation and Analysis of the Decomposition-Fusion ECCM Technique. In Proc. 2000 SPIE Conf. on Signal and Data Processing of Small Targets, vol. 4048, pages 486-497, Orlando, Florida, USA, April 2000.

[26] P. Smets and B. Ristic. Kalman Filter and Joint Tracking and Classification in the TBM Framework. In Proc. 7th International Conf. on Information Fusion, volume I, pages 46-53, Stockholm, Sweden, June 28-July 12004.

[27] S. Tsai, T.F. Wong, and J.S. Lehnert. DS-CDMA System with Joint Channel Estimation and MAP Detection in Time-Selective Fading Channels. IEEE Journal on Selected Areas in Communications, 19(1):121$131,2001$.

[28] B. Vo and W.-K. Ma. Joint Detection and Tracking of Multiple Maneuvering Targets in Clutter Using Random Finite Sets. In Proc. 2004 Control, Automation, Robotics and Vision Conference, volume 2, pages 1485-1490, Dec. 2004. 